\title{
Parameter identification on flexible multibody models using the adjoint variable method and flexible natural coordinate formulation
}

\author{
Simon Vanpaemel ${ }^{* i}$ \\ DMMS lab \\ Flanders Make \\ Department of Mechanical Engineering \\ KU Leuven \\ Celestijnenlaan 300 \\ Leuven, 3000 \\ Belgium \\ Email: simon.vanpaemel@kuleuven.be \\ Frank Naets \\ DMMS lab \\ Flanders Make \\ Department of Mechanical Engineering \\ KU Leuven \\ Celestijnenlaan 300 \\ Leuven, 3000 \\ Belgium \\ Email: frank.naets@kuleuven.be \\ Martijn Vermaut \\ DMMS lab \\ Flanders Make \\ Department of Mechanical Engineering \\ KU Leuven \\ Celestijnenlaan 300 \\ Leuven, 3000 \\ Belgium \\ Email: martijn.vermaut@kuleuven.be \\ Wim Desmet \\ DMMS lab \\ Flanders Make \\ Department of Mechanical Engineering \\ KU Leuven \\ Celestijnenlaan 300 \\ Leuven, 3000 \\ Belgium \\ Email:wim.desmet@kuleuven.be
}




\section{ABSTRACT}

This work proposes a methodology for in-situ parameter identification using system-level measurements of (flexible) multibody systems (FMBS), opposed to dedicated component-level identification. The sensitivity information employed for the optimization is obtained using the Adjoint Variable Method (AVM). This method has the advantage of obtaining sensitivity information at a computational cost independent of the amount of model parameters. The underlying flexible multibody formulation employed is a novel approach called the flexible natural coordinates formulation (FNCF). This formulation combines the advantageous properties of the floating frame of reference formulation (FFRF) and the generalized component mode synthesis (GCMS) methods and results in a constant mass and stiffness matrix with quadratic constraint equations. This work shows how the specific structure of equations obtained through FNCF drastically reduces the complexity of the AVM as the simulation derivatives can be readily obtained and are of limited order. The proposed approach has been implemented in an in-house object-oriented Matlab multibody code. The methodology is illustrated by identifying thirteen model parameters of a MacPherson suspension model, in-situ and using system-level measurements.

\section{Introduction}

Mechatronic systems of industrial complexity generally include complex, nonlinear components such as bushings, dampers, non-ideal joints, etc... Because these elements have a high impact on the dynamic loads and vibrations transferred through the system, models of these parts should often be included in multibody simulations in order to reach the desired accuracy. However, in current practice the characterization of these components for operationally representative boundary conditions and load cases is lacking. Experimental parameter identification methods exist, but these are typically limited to isolated identification on dedicated test rigs [1-3], such that their in-situ representatives is often limited. Therefore, the authors focus on parameter identification techniques that can be applied on a system level.

Optimization routines often employ sensitivity information in order to obtain faster convergence [4]. However, the generation of this information comes at a cost, which depends on the employed sensitivity method. There exist a variety of methods, of which finite differences (FD), the direct differentiation method (DDM) [5,6], the AVM [6-8] and automatic differentiation (AD) [9] are most notable. The efficiency and accuracy of these methods with respect to each other depend heavily on the problem that needs to be optimized. The authors propose to exploit the AVM method for obtaining the sensitivity information, because using this approach the computational complexity is relatively unaffected by the amount of model parameters, which makes it very well suited for problems with a large design space. The AVM requires backward integration in time of the adjoint equations, which are described by differential algebraic equations (DAE). The use of a (flexible) multibody formulation for which terms in the adjoint equations drop out, or are of low complexity to evaluate, reduces the computational cost of the sensitivity information. It is therefore proposed to exploit the FNCF, recently introduced by Vermaut et al. [10]. The specific structure of equations obtained through FNCF drastically reduces the complexity of the AVM as the simulation derivatives can be readily obtained and are of limited order.

An important aspect related to parameter identification exercises, is the identifiability of the design parameters. Although important, for sake of brevity this will not be the focus of this manuscript. The interested reader is referred to other works such as $[5,11,12]$.

The paper is organised as follows. Section 2 discusses an overview of the parameter identification. Section 3 presents a brief summary of the flexible natural coordinates multibody formulation. Section 4 focuses on applying this formulation with the AVM. Section 5 illustrates this methodology on the identification of the parameters of a suspension strut and discusses the corresponding results. Finally, Section 6 concludes this work.

\section{Parameter identification of multibody models}

Parameter identification is an optimization process that minimizes an objective function for a number of model parameters $\boldsymbol{\rho}$. In this work the objective function $\psi$ is the integrand of a least squares error $g$ between a simulated quantity $y$ and a measured quantity $\hat{y}$ during an user defined time window starting from $T_{0}$ and ending at $T_{F}$ :

$$
\psi=\int_{T_{0}}^{T_{F}} g(\mathbf{q}, \dot{\mathbf{q}}, \ddot{\mathbf{q}}, \boldsymbol{\lambda}, \boldsymbol{\rho}) \mathrm{dt}=\int_{\mathrm{T}_{0}}^{\mathrm{T}_{\mathrm{F}}}(\mathrm{y}(\mathbf{q}, \dot{\mathbf{q}}, \ddot{\mathbf{q}}, \boldsymbol{\lambda}, \boldsymbol{\rho})-\hat{\mathrm{y}}(\mathrm{t}))^{2} \mathrm{dt} .
$$

Furthermore, it is assumed that the objective function has no specific constraints at time $T_{F}$. The simulated quantities depend in general on the generalized coordinates $\mathbf{q}=\left[\begin{array}{llll}\mathrm{q}_{1} & \mathrm{q}_{2} & \cdots & \mathrm{q}_{\mathrm{q}}\end{array}\right] \in \mathbb{R}^{n_{q}}$, velocities $\dot{\mathbf{q}} \in \mathbb{R}^{n_{q}}$, accelerations $\ddot{\mathbf{q}} \in \mathbb{R}^{n_{q}}$, Lagrange multipliers $\lambda \in \mathbb{R}^{n_{\lambda}}$ and the model parameters $\boldsymbol{\rho} \in \mathbb{R}^{n_{\rho}}$. Where $\mathbf{q}, \dot{\mathbf{q}}, \ddot{\mathbf{q}}, \boldsymbol{\lambda}$ are determined by the solution of the multibody 
systems' equations of motion (EOM) $\mathbf{h}$ and constraint equations $\boldsymbol{\phi}$ :

$$
\left\{\begin{array}{l}
\mathbf{h}(\mathbf{q}, \dot{\mathbf{q}}, \ddot{\mathbf{q}}, \lambda, \boldsymbol{\rho})=\mathbf{0} \\
\boldsymbol{\phi}(\mathbf{q}, \boldsymbol{\rho})=\mathbf{0}
\end{array}\right.
$$

In this work the subscript []$_{T_{0}}$ denotes the evaluation at time $T_{0}$, the subscript []$_{T_{F}}$ the evaluation of the variables at time $T_{F}$ and the operator $\left(\dot{)}\right.$ the time derivative of that specific variable. Furthermore, in this work it is assumed that time $T_{0}$ and $T_{F}$ are fixed.

\subsection{Sensitivity analysis}

Sensitivity analysis provides us with information on how the objective function changes due to a change in the model parameters. Sensitivity information can be obtained by different methods, of which finite differences (FD), direct differentiation method (DDM), adjoint variable method (AVM) and automatic differentiation (AD) are the most notable. The advantages and disadvantages of each method are briefly discussed in the following sections.

\subsubsection{Finite differences}

The finite difference approximation is a numerical method where the sensitivity is obtained by small perturbations of each model parameter. In the case of a first order forward differentiation the sensitivity is defined as

$$
\frac{\mathrm{d} \psi}{\mathrm{d} \rho_{\mathrm{i}}} \approx \frac{\left(\psi\left(\boldsymbol{\rho}+\delta_{p} \mathbf{e}_{i}\right)-\psi(\boldsymbol{\rho})\right)}{\delta_{p}}, \quad \text { for } \mathrm{i}=1, \ldots, n_{\rho}
$$

with $\delta_{p} \mathbf{e}_{i}$ a small perturbation of the i-th model parameter. The advantage of this method is the very limited and straightforward implementation to obtain sensitivity information. However, the downside of this method is (i) the accuracy depends on the perturbation step-size $\delta_{p}$, (ii) even for an optimal perturbation step the results for the sensitivity are less accurate than the function evaluation itself, (iii) for each model parameter a forward simulation must be performed [13].

\subsubsection{Direct differentiation method}

The direct differentiation method obtains the sensitivity information by taking the variation of the objective function $\psi$ :

$$
\delta \psi=\int_{T_{0}}^{T_{F}}\left(\frac{\partial g}{\partial \mathbf{q}} \delta \mathbf{q}+\frac{\partial g}{\partial \dot{\mathbf{q}}} \delta \dot{\mathbf{q}}+\frac{\partial g}{\partial \ddot{\mathbf{q}}} \boldsymbol{\delta} \ddot{\mathbf{q}}+\frac{\partial g}{\partial \boldsymbol{\lambda}} \boldsymbol{\delta} \boldsymbol{\lambda}+\frac{\partial g}{\partial \boldsymbol{\rho}} \boldsymbol{\delta} \boldsymbol{\rho}\right) \mathrm{dt}
$$

The terms which are not readily available or computable are the variations of the state variables, that are defined as follows:

$$
\delta \mathbf{q}=\frac{\mathrm{d} \mathbf{q}}{\mathrm{d} \rho} \delta \boldsymbol{\rho}, \quad \delta \dot{\mathbf{q}}=\frac{\mathrm{d} \dot{\mathbf{q}}}{\mathrm{d} \rho} \delta \rho, \quad \delta \ddot{\mathbf{q}}=\frac{\mathrm{d} \ddot{\mathbf{q}}}{\mathrm{d} \rho} \delta \rho, \quad \delta \lambda=\frac{\mathrm{d} \lambda}{\mathrm{d} \rho} \delta \rho
$$

The derivatives of the state variables $\frac{\mathrm{d} \mathbf{q}}{\mathrm{d} \boldsymbol{\rho}}, \frac{\mathrm{d} \dot{\mathbf{q}}}{\mathrm{d} \boldsymbol{\rho}}, \frac{\mathrm{d} \ddot{\mathbf{q}}}{\mathrm{\rho} \boldsymbol{\rho}}$ and $\frac{\mathrm{d} \lambda}{\mathrm{d} \boldsymbol{\rho}}$ are obtained from the integration of the differentiated equations of motion with respect to the model parameters, and using the initial conditions $\left[\frac{\mathrm{d} \mathbf{q}}{\mathrm{d} \boldsymbol{\rho}}, \frac{\mathrm{d} \dot{\mathbf{q}}}{\mathrm{d} \boldsymbol{\rho}}, \frac{\mathrm{d} \ddot{\mathbf{q}}}{\mathrm{d} \boldsymbol{\rho}}, \frac{\mathrm{d} \boldsymbol{\lambda}}{\mathrm{d} \boldsymbol{\rho}}\right]_{T_{0}}[6]$. These equations are solved simultaneously with the equations of motion. This results in the disadvantage that the computational cost of the gradient computation scales directly with the amount of model parameters. The advantage of this method is that the sensitivity information is exact and is obtained with the same accuracy as the objective function evaluation [7].

\subsubsection{Automatic differentiation}

Automatic differentiation computes the sensitivity information by applying the chain rule of differentiation repeatedly to the performed operations. Typically AD can be divided into a forward and reverse method. The forward method scales with the amount of model parameters, but not with the amount of objective functions. The reverse method scales with the amount of objective functions, but not with the amount of model parameters. The advantages are that the sensitivity information is computed up to machine precision and, depending on the platform, the limited implementation complexity due to the available toolboxes. The disadvantage is the immense data storage needed in case of the reverse method [4]. 


\subsubsection{Adjoint variable method}

The adjoint variable method adds the equations of motion, defined in Eq.(2), to the objective function using Lagrange multipliers $\boldsymbol{\mu}$ and $\boldsymbol{\mu}_{\phi}$, which are also called adjoint variables. The equations of motion must be fulfilled at every instance of time between $T_{0}$ and $T_{F}$ :

$$
\psi=\int_{T_{0}}^{T_{F}} g \mathrm{dt}-\int_{\mathrm{T}_{0}}^{\mathrm{T}_{\mathrm{F}}} \boldsymbol{\mu}^{\mathrm{T}} \mathbf{h} \mathrm{dt}-\int_{\mathrm{T}_{0}}^{\mathrm{T}_{\mathrm{F}}} \boldsymbol{\mu}_{\boldsymbol{\phi}}^{\mathrm{T}} \boldsymbol{\phi} \mathrm{dt}
$$

Variation of the objective function and applying partial integration in order to rewrite $\delta \dot{\mathbf{q}}$ and $\delta \ddot{\mathbf{q}}$ in terms of $\delta \mathbf{q},[\delta \mathbf{q}, \delta \dot{\mathbf{q}}]_{T_{0}}$ and $[\delta \mathbf{q}, \delta \dot{\mathbf{q}}]_{T_{F}}$, gives:

$$
\begin{aligned}
\delta \psi= & \int_{T_{0}}^{T_{F}}\left(\frac{\partial g}{\partial \mathbf{q}}-\boldsymbol{\mu}^{\mathrm{T}} \frac{\partial \mathbf{h}}{\partial \mathbf{q}}-\boldsymbol{\mu}_{\boldsymbol{\phi}}^{\mathrm{T}} \frac{\partial \boldsymbol{\phi}}{\partial \mathbf{q}}\right) \delta \mathbf{q} \mathrm{dt}+\left[\left(\frac{\partial \mathrm{g}}{\partial \dot{\mathbf{q}}}-\boldsymbol{\mu}^{\mathrm{T}} \frac{\partial \mathbf{h}}{\partial \dot{\mathbf{q}}}\right) \delta \mathbf{q}\right]_{\mathrm{T}_{\mathrm{F}}}-\left[\left(\frac{\partial \mathrm{g}}{\partial \dot{\mathbf{q}}}-\boldsymbol{\mu}^{\mathrm{T}} \frac{\partial \mathbf{h}}{\partial \dot{\mathbf{q}}}\right) \delta \mathbf{q}\right]_{\mathrm{T}_{0}} \\
& -\int_{T_{0}}^{T_{F}}\left(\frac{\mathrm{d}}{\mathrm{dt}}\left(\frac{\partial \mathrm{g}}{\partial \dot{\mathbf{q}}}\right)-\dot{\boldsymbol{\mu}}^{\mathrm{T}} \frac{\partial \mathbf{h}}{\partial \dot{\mathbf{q}}}-\boldsymbol{\mu}^{\mathrm{T}} \frac{\mathrm{d}}{\mathrm{dt}}\left(\frac{\partial \mathbf{h}}{\partial \dot{\mathbf{q}}}\right)\right) \delta \mathbf{q} \mathrm{dt}+\left[\left(\frac{\partial \mathrm{g}}{\partial \ddot{\mathbf{q}}}-\boldsymbol{\mu}^{\mathrm{T}} \frac{\partial \mathbf{h}}{\partial \ddot{\mathbf{q}}}\right) \delta \dot{\mathbf{q}}\right]_{\mathrm{T}_{\mathrm{F}}}-\left[\left(\frac{\partial \mathrm{g}}{\partial \ddot{\mathbf{q}}}-\boldsymbol{\mu}^{\mathrm{T}} \frac{\partial \mathbf{h}}{\partial \ddot{\mathbf{q}}}\right) \delta \dot{\mathbf{q}}\right]_{\mathrm{T}_{0}} \\
& -\left[\left(\frac{\mathrm{d}}{\mathrm{dt}}\left(\frac{\partial g}{\partial \ddot{\mathbf{q}}}\right)-\dot{\boldsymbol{\mu}}^{\mathrm{T}} \frac{\partial \mathbf{h}}{\partial \ddot{\mathbf{q}}}-\boldsymbol{\mu}^{\mathrm{T}} \frac{\mathrm{d}}{\mathrm{dt}}\left(\frac{\partial \mathbf{h}}{\partial \ddot{\mathbf{q}}}\right)\right) \delta \mathbf{q}\right]_{T_{F}}+\left[\left(\frac{\mathrm{d}}{\mathrm{dt}}\left(\frac{\partial g}{\partial \ddot{\mathbf{q}}}\right)-\dot{\boldsymbol{\mu}}^{\mathrm{T}} \frac{\partial \mathbf{h}}{\partial \ddot{\mathbf{q}}}-\boldsymbol{\mu}^{\mathrm{T}} \frac{\mathrm{d}}{\mathrm{dt}}\left(\frac{\partial \mathbf{h}}{\partial \ddot{\mathbf{q}}}\right)\right) \delta \mathbf{q}\right]_{T_{0}} \\
& +\int_{T_{0}}^{T_{F}}\left(\frac{\mathrm{d}^{2}}{\mathrm{dt}^{2}}\left(\frac{\partial g}{\partial \ddot{\mathbf{q}}}\right)-\ddot{\boldsymbol{\mu}}^{\mathrm{T}} \frac{\partial \mathbf{h}}{\partial \ddot{\mathbf{q}}}-2 \dot{\boldsymbol{\mu}}^{\mathrm{T}} \frac{\mathrm{d}}{\mathrm{dt}}\left(\frac{\partial \mathbf{h}}{\partial \ddot{\mathbf{q}}}\right)-\boldsymbol{\mu}^{\mathrm{T}} \frac{\mathrm{d}^{2}}{\mathrm{dt}^{2}}\left(\frac{\partial \mathbf{h}}{\partial \ddot{\mathbf{q}}}\right)\right) \delta \mathbf{q} \mathrm{dt} \\
& +\int_{T_{0}}^{T_{F}}\left(\frac{\partial g}{\partial \lambda}-\boldsymbol{\mu}^{\mathrm{T}} \frac{\partial \mathbf{h}}{\partial \boldsymbol{\lambda}}\right) \delta \boldsymbol{\lambda} \mathrm{dt}+\int_{\mathrm{T}_{0}}^{\mathrm{T}_{\mathrm{F}}}\left(\frac{\partial \mathrm{g}}{\partial \boldsymbol{\rho}}-\boldsymbol{\mu}^{\mathrm{T}} \frac{\partial \mathbf{h}}{\partial \boldsymbol{\rho}}-\boldsymbol{\mu}_{\phi}^{\mathrm{T}} \frac{\partial \boldsymbol{\phi}}{\partial \boldsymbol{\rho}}\right) \delta \boldsymbol{\rho} \mathrm{dt} .
\end{aligned}
$$

Key in this method is to compute the adjoint variables that eliminate the coefficients of $\delta \mathbf{q}$ and $\delta \boldsymbol{\lambda}$. In this way the need for calculating $\delta \mathbf{q}$ and $\delta \boldsymbol{\lambda}$, which is done using the direct differentiation method, is circumvented. This results in a system of differential algebraic equations (DAE) of index three, called the adjoint equations, to be solved backwards in time for the adjoint variables $\boldsymbol{\mu}$ and $\boldsymbol{\mu}_{\phi}$. Furthermore, the 'initial conditions' for these equations at time $T_{F}$ are obtained by the elimination of $[\mathbf{q}, \dot{\mathbf{q}}, \boldsymbol{\lambda}]_{T_{F}}$, for more information the reader is referred to [14].

The main advantages of the AVM is that it generates highly accurate sensitivity information, and that the computational cost of solving the adjoint system is relatively independent from the amount of model parameters. The disadvantage of this method is an error-prone implementation, and the data storage needed for the state variables which are obtained during the forward simulation. Furthermore, the computational cost scales with the number of objective functions to be minimized.

\section{Flexible Natural Coordinates Formulation (FNCF)}

FNCF is a small deformation flexible multibody formulation that results in a simple structure of equations of having constant mass and stiffness matrices with quadratic constraint equations. Moreover, no quadratic velocity term appears as is the case when using a floating frame of reference (FFR) multibody formulation, nor a term which is nonlinear in the flexible deformation as is the case when using a generalized component mode synthesis (GCMS) multibody formulation. The cost of obtaining this structure of equations is the introduction of redundancy in the set of generalized coordinates. This work summarizes the main concepts of the FNCF approach which will be exploited to set up the AVM parameter identification framework. For further details on this method the interested reader is referred to [10].

\subsection{Kinematics of a single body}

The generalized coordinate vector $\mathbf{q}=[\boldsymbol{\sigma} \boldsymbol{\pi} \boldsymbol{\gamma} \boldsymbol{\delta}]^{\mathrm{T}}$ used in this and the following Section 3.2 contains only the generalized coordinates associated with a single flexible body. The assembled system is considered from Section 3.3 onwards. The flexible body is represented by a finite element (FE) model that is described with a stiffness matrix $\mathbf{K}^{\mathrm{FE}} \in \mathbb{R}^{\mathrm{N} \times \mathrm{N}}$ and lumped mass matrix $\mathbf{M}^{\mathrm{FE}} \in \mathbb{R}^{\mathrm{N} \times \mathrm{N}}$. The generalized coordinate vector of a single body consists of 4 'types' of coordinates. First, $\boldsymbol{\sigma} \in \mathbb{R}^{3}$ which defines the position of the reference frame of the body. Second, $\boldsymbol{\pi} \in \mathbb{R}^{9}$ are redundant coordinates that parametrize the rotation matrix of the body. Third, $\boldsymbol{\delta} \in \mathbb{R}^{n_{\delta}}$ are the modal participation factors of the modes $\Psi \in \mathbb{R}^{\mathrm{N} \times n_{\delta}}$ used to reduce the FE model, with $n_{\delta}$ the amount of modes. These participation factors describe the deformation in the bodyfixed reference frame, as in the FFR multibody formulation. Fourth, the FNCF introduces new, but redundant, coordinates $\gamma$ $\in \mathbb{R}^{9 n_{\delta}}$, computed from $\boldsymbol{\pi}$ and $\boldsymbol{\delta}$ using a Kronecker product:

$$
\boldsymbol{\gamma}=\boldsymbol{\delta} \otimes \boldsymbol{\pi}
$$


These coordinates enable a linear relationship between the generalized coordinates $\mathbf{q}$ and the position $\mathbf{u}^{(p)}$ of a point $p$ on a deformable body, using a constant projection matrix $\mathbf{r}_{\mathbf{q}}^{(p)} \in \mathbb{R}^{3 \times\left(12+10 n_{\delta}\right)}$ :

$$
\mathbf{u}^{(p)}=\left[\begin{array}{llll}
\mathbf{I}_{3} & \mathbf{r}_{\boldsymbol{\pi}}^{(p)} & \mathbf{r}_{\boldsymbol{\gamma}}^{(p)} & \mathbf{0}_{3 \times \mathrm{n}_{\delta}}
\end{array}\right]\left[\begin{array}{c}
\boldsymbol{\sigma} \\
\boldsymbol{\pi} \\
\boldsymbol{\gamma} \\
\boldsymbol{\delta}
\end{array}\right]=\mathbf{r}_{\mathbf{q}}^{(p)} \mathbf{q} \quad \Rightarrow \quad\left\{\begin{array}{l}
\dot{\mathbf{u}}^{(p)}=\mathbf{r}_{\mathbf{q}}^{(p)} \dot{\mathbf{q}} \\
\ddot{\mathbf{u}}^{(p)}=\mathbf{r}_{\mathbf{q}}^{(p)} \ddot{\mathbf{q}}
\end{array}\right.
$$

The rotation matrix of a point $p$, describing rigid body rotation and the rotation due to the small deformation, can be written using the linear combination:

$$
\mathbf{R}^{(p)}=\sum_{i=1}^{9} \mathbf{P}_{i} \pi_{i}+\sum_{i=1}^{9 n_{\delta}} \mathbf{R}_{\gamma, i}^{(p)} \gamma_{i}
$$

wherein $\mathbf{P}_{i} \in \mathbb{R}^{3 \times 3}$ and $\mathbf{R}_{\gamma, i}^{(p)} \in \mathbb{R}^{3 \times 3}$ are both constant shape matrices and $\pi_{i}$ and $\gamma_{i}$ are scalar values representing the $i^{t h}$ index of $\boldsymbol{\pi}$ and $\boldsymbol{\gamma}$ respectively. This results in a rotation matrix being linear in the generalized coordinates $\mathbf{q}$. This is not the case using another multibody formulation such as FFR or GCMS, of which the rotation matrix is nonlinear in the generalized coordinates.

\subsection{Dynamics of a single body}

The deformations of the flexible bodies are assumed small, such that the linear finite element (FE) mass and stiffness matrices, $\mathbf{M}^{\mathrm{FE}}$ and $\mathbf{K}^{\mathrm{FE}}$ respectively, are constant. It is assumed that $\mathbf{M}^{\mathrm{FE}}$ is a lumped mass matrix. The FE mass matrix can be reduced to the reduced generalized mass matrix $\mathbf{M}_{\mathbf{q q}}$ using the constant projection matrix $\mathbf{r}_{\mathbf{q}}$ :

$$
\mathbf{M}_{\mathbf{q q}}=\mathbf{r}_{\mathbf{q}}^{\mathrm{T}} \mathbf{M}^{\mathrm{FE}} \mathbf{r}_{\mathbf{q}}
$$

this matrix is a generalization of the projection matrix $\mathbf{r}_{\mathbf{q}}^{(p)}$, for all nodes in the FE model. Note that the reduced mass matrix is constant and thus independent of the generalized coordinates $\mathbf{q}$. Because the projection matrix $\mathbf{r}_{\mathbf{q}}$ is nonlinear in the case of the FFR multibody formulation, it ends up with a nonlinear reduced mass matrix [15].

The computation of the reduced stiffness matrix $\mathbf{K}_{\mathbf{q q}}$ is similar to the FFR multibody formulation:

$$
\mathbf{K}_{\mathbf{q q}}=\left[\begin{array}{c}
\mathbf{0}_{\left(12+9 n_{\delta}\right) \times \mathrm{N}} \\
\boldsymbol{\Psi}^{\mathrm{T}}
\end{array}\right] \mathbf{K}^{\mathrm{FE}}\left[\mathbf{0}_{\mathrm{N} \times\left(12+9 n_{\delta}\right)} \boldsymbol{\Psi}\right]
$$

where the matrix is constant and thus independent of the generalized coordinates $\mathbf{q}$. The GCMS formulation on the other hand ends up with a nonlinear reduced stiffness matrix, since these local coordinates are not included in the generalized coordinate vector [16].

This illustrates the reason for introducing redundant flexible coordinates $\boldsymbol{\delta}$ and $\boldsymbol{\gamma}$ in the generalized coordinate vector q. Because the 'FFR' flexible coordinates $\boldsymbol{\delta}$ are used to derive the constant reduced stiffness matrix, while the 'GCMS' coordinates $\boldsymbol{\sigma}, \boldsymbol{\pi}$ and $\boldsymbol{\gamma}$ are used to derive the constant reduced mass matrix.

\subsection{FNCF constraints}

The constraint equations of a (flexible) multibody systems can be divided into two groups. The first group apply to a single (flexible) body, e.g. the orthonormality constraints of the body's rotation matrix and the introduction of the redundant coordinates $\boldsymbol{\gamma}$, the latter given by Eq.(8), which are both quadratic in the generalized coordinates. The second group are applied between different (flexible) components. These constraints are often called joint constraints and are assumed to be holonomic in this work. They enable the modelling of ideal joints. In FNCF, the larger part of these constraints can be expressed as a linear or quadratic function of the generalized coordinates as well.

Since the complete set of constraint equations are at most quadratic in the generalized coordinates, it is possible to write the constraint equations as a Taylor expansion around $\mathbf{q}=\mathbf{0}$ :

$$
\boldsymbol{\phi}=\mathbf{C}_{0}+\mathbf{J}_{0} \mathbf{q}+\frac{1}{2}\left(\sum_{i} \mathbf{H}_{0, i} \mathbf{q}_{i}\right) \mathbf{q}=\mathbf{0}
$$


wherein $\mathbf{J}_{0}$ and $\mathbf{H}_{0, i}$ are constant matrices.

\subsection{Equations of motion}

The reduced mass matrix $\mathbf{M}_{\mathbf{q q}}$ and stiffness matrix $\mathbf{K}_{\mathbf{q q}}$ of the flexible bodies can now be assembled in the mass and stiffness matrix of the complete multibody system, denoted as $\mathbf{M}$ and $\mathbf{K}$ respectively. The fully assembled equations of motion can be summarized as:

$$
\left\{\begin{array}{l}
\mathbf{M} \ddot{\mathbf{q}}+\frac{\partial \boldsymbol{\phi}^{\mathrm{T}}}{\partial \mathbf{q}} \boldsymbol{\lambda}=\mathbf{f} \\
\boldsymbol{\phi}=\mathbf{0}
\end{array}\right.
$$

with:

$$
\mathbf{f}=\mathbf{f}_{g r a}-\mathbf{C} \dot{\mathbf{q}}-\mathbf{K q}+\mathbf{f}_{e x t}+\mathbf{f}_{i n t}
$$

where $\mathbf{f}_{\text {ext }}$ is the generalized force vector of the externally applied loads, $\mathbf{f}_{i n t}$ is the generalized force vector of the internal loads acting between the components. The reduced damping matrix is denoted as $\mathbf{C}$, which is constant for Rayleigh damping on the deformations. The generalized force vector $\mathbf{f}_{\text {gra }}$ of the gravity force is constant because of the constant projection matrix $\mathbf{r}_{\mathbf{q}}$, it can be written as follows: $\mathbf{f}_{g r a}=\mathbf{r}_{\mathbf{q}}^{\mathrm{T}} \mathbf{M}^{\mathrm{FE}} \mathbf{g}$, with $\mathbf{g}$ the gravitational acceleration for each mode.

Note that the projection matrices between the external loads applied in a local or global frame $\boldsymbol{v}_{\text {ext }}$ and the generalized external force vector $\mathbf{f}_{\text {ext }}$ are constant or linear in the generalized coordinates. This is not the case when using either a FFR or GCMS multibody formulation, since these end up with complex nonlinear projection matrices.

\section{Adjoint Variable Method using FNCF}

The properties of FNCF will now be applied to the adjoint equations derived for a general multibody formulation, obtained from [14]. The simple structure of equations of FNCF results in the elimination of $\ddot{\mathbf{M}}, \dot{\mathbf{M}}$ and $\frac{\partial \mathbf{M}}{\partial \mathbf{q}}$ in the adjoint equations. These terms do appear in the equations when using FFRF. The resulting adjoint system reduces to the following set of equations:

$$
\left\{\begin{array}{l}
\mathbf{M}^{\mathrm{T}} \ddot{\boldsymbol{\mu}}+\frac{\partial \mathbf{f}^{\mathrm{T}}}{\partial \dot{\mathbf{q}}} \dot{\boldsymbol{\mu}}+\left(\frac{\partial^{2} \boldsymbol{\phi}^{\mathrm{T}}}{\partial \mathbf{q} \partial \mathbf{q}} \otimes \lambda-\frac{\partial \mathbf{f}}{\partial \mathbf{q}}+\frac{\mathrm{d}}{\mathrm{dt}}\left(\frac{\partial \mathbf{f}}{\partial \dot{\mathbf{q}}}\right)\right)^{\mathrm{T}} \boldsymbol{\mu}+\frac{\partial \boldsymbol{\phi}^{\mathrm{T}}}{\partial \mathbf{q}} \boldsymbol{\mu}_{\boldsymbol{\phi}}=\frac{\partial \mathrm{g}}{\partial \mathbf{q}}-\frac{\mathrm{d}}{\mathrm{dt}}\left(\frac{\partial \mathrm{g}}{\partial \dot{\mathbf{q}}}\right)+\frac{\mathrm{d}^{2}}{\mathrm{dt}^{2}}\left(\frac{\partial \mathrm{g}}{\partial \ddot{\mathbf{q}}}\right) \\
\frac{\partial \boldsymbol{\phi}}{\partial \mathbf{q}} \boldsymbol{\mu}=\frac{\partial \mathrm{g}}{\partial \boldsymbol{\lambda}}
\end{array}\right.
$$

that must be solved backwards in time for the adjoint variables $\boldsymbol{\mu}$ and $\boldsymbol{\mu}_{\phi}$, by using the 'initial' conditions at time $T_{F}$ which are obtained from solving:

$$
\begin{aligned}
& {\left[\mathbf{M}^{\mathrm{T}} \dot{\boldsymbol{\mu}}+\frac{\partial \mathbf{f}^{\mathrm{T}}}{\partial \dot{\mathbf{q}}} \boldsymbol{\mu}+\frac{\partial \boldsymbol{\phi}^{\mathrm{T}}}{\partial \mathbf{q}} \boldsymbol{\gamma}+\left(\frac{\partial^{2} \boldsymbol{\phi}}{\partial \mathbf{q} \partial \mathbf{q}} \otimes \dot{\mathbf{q}}+\frac{\partial^{2} \boldsymbol{\phi}}{\partial \mathbf{q} \partial \mathrm{t}}\right)^{\mathrm{T}} \boldsymbol{\eta}\right]_{T_{F}} }=\left[\frac{\mathrm{d}}{\mathrm{dt}}\left(\frac{\partial \mathrm{g}}{\partial \ddot{\mathbf{q}}}\right)-\left(\frac{\partial \mathrm{g}}{\partial \dot{\mathbf{q}}}\right)\right]_{T_{F}} \\
& {\left[\mathbf{M}^{\mathrm{T}} \boldsymbol{\mu}+\frac{\partial \boldsymbol{\phi}^{\mathrm{T}}}{\partial \mathbf{q}} \boldsymbol{\eta}\right]_{T_{F}}=} {\left[\frac{\partial \mathrm{g}}{\partial \ddot{\mathbf{q}}}\right]_{T_{F}} } \\
& {\left[\frac{\partial \boldsymbol{\phi}}{\partial \mathbf{q}} \boldsymbol{\mu}\right]_{T_{F}} }=\left[\frac{\partial \mathrm{g}}{\partial \boldsymbol{\lambda}}\right]_{T_{F}} \\
& {\left[\frac{\partial \boldsymbol{\phi}}{\partial \mathbf{q}} \dot{\boldsymbol{\mu}}+\frac{\partial \dot{\boldsymbol{\phi}}}{\partial \mathbf{q}} \boldsymbol{\mu}\right]_{T_{F}}=\left[\frac{\mathrm{d}}{\mathrm{dt}}\left(\frac{\partial \mathrm{g}}{\partial \boldsymbol{\lambda}}\right)\right]_{T_{F}} }
\end{aligned}
$$


The sensitivity of the objective function $\psi$ w.r.t. the model parameters $\rho$ is given by:

$$
\begin{array}{r}
\nabla_{\boldsymbol{\rho}} \psi^{\mathrm{T}}=\left[\left(\frac{\mathrm{d}}{\mathrm{dt}}\left(\frac{\partial \mathrm{g}}{\partial \ddot{\mathbf{q}}}\right)-\frac{\partial \mathrm{g}}{\partial \dot{\mathbf{q}}}-\dot{\boldsymbol{\mu}}^{\mathrm{T}} \mathbf{M}-\boldsymbol{\mu}^{\mathrm{T}} \frac{\partial \mathbf{f}}{\partial \dot{\mathbf{q}}}\right) \frac{\mathrm{d} \mathbf{q}}{\mathrm{d} \boldsymbol{\rho}}\right]_{T_{0}}-\left[\left(\frac{\partial \mathrm{g}}{\partial \ddot{\mathbf{q}}}-\boldsymbol{\mu}^{\mathrm{T}} \mathbf{M}\right) \frac{\mathrm{d} \dot{\mathbf{q}}}{\mathrm{d} \boldsymbol{\rho}}\right]_{T_{0}} \\
+\int_{T_{0}}^{T_{F}}\left(\frac{\partial \mathrm{g}}{\partial \boldsymbol{\rho}}-\boldsymbol{\mu}^{\mathrm{T}}\left(\frac{\partial \mathbf{M}}{\partial \boldsymbol{\rho}} \otimes \ddot{\mathbf{q}}+\frac{\partial^{2} \boldsymbol{\phi}^{\mathrm{T}}}{\partial \mathbf{q} \partial \boldsymbol{\rho}} \otimes \boldsymbol{\lambda}-\frac{\partial \mathbf{f}}{\partial \boldsymbol{\rho}}\right)-\boldsymbol{\mu}_{\boldsymbol{\phi}}^{\mathrm{T}} \frac{\partial \boldsymbol{\phi}}{\partial \boldsymbol{\rho}}\right) \mathrm{dt}
\end{array}
$$

The terms $\left[\frac{\mathrm{d} \mathbf{q}}{\mathrm{d} \boldsymbol{\rho}}, \frac{\mathrm{d} \dot{\mathbf{q}}}{\mathrm{\rho} \boldsymbol{\rho}}\right]_{T_{0}}$ are obtained through direct differentiation of the equations of motion and its initial conditions at time $T_{0}[6]$.

\subsection{Derivatives of the constraint equations}

Because the constraint equations, defined in Eq.(13), are quadratic in the generalized coordinates, the first order partial derivative with respect to the generalized coordinates $\mathbf{q}$ results in a linear dependence of the generalized coordinates. Similarly, the second order partial derivatives result in a constant matrix:

$$
\frac{\partial \boldsymbol{\phi}}{\partial \mathbf{q}}=\mathbf{J}_{0}+\left(\sum_{\mathrm{i}} \mathbf{H}_{0, \mathrm{i}} \mathbf{q}_{\mathrm{i}}\right)
$$

$$
\frac{\partial^{2} \boldsymbol{\phi}}{\partial \mathbf{q}_{\mathrm{i}} \partial \mathbf{q}}=\mathbf{H}_{0, \mathrm{i}} \quad \mathrm{i}=1,2, \ldots, \mathrm{n}_{\mathrm{q}}
$$

\subsection{Derivatives of the generalized forces}

This section focuses on the derivatives of the terms in the generalized force vector. The following derivatives appear in the adjoint equations:

$$
\begin{aligned}
& \mathbf{f}_{\mathbf{q}}=\frac{\partial \mathbf{f}}{\partial \mathbf{q}}=-\mathbf{K}+\frac{\partial \mathbf{f}_{e x t}}{\partial \mathbf{q}}+\frac{\partial \mathbf{f}_{i n t}}{\partial \mathbf{q}} \\
& \mathbf{f}_{\dot{q}}=\frac{\partial \mathbf{f}}{\partial \dot{\mathbf{q}}}=-\mathbf{C}+\frac{\partial \mathbf{f}_{i n t}}{\partial \dot{\mathbf{q}}}
\end{aligned}
$$

$$
\begin{aligned}
& \dot{\mathbf{f}}_{\dot{\mathbf{q}}}=\frac{\mathrm{d}}{\mathrm{dt}}\left(\frac{\partial \mathbf{f}_{i n t}}{\partial \dot{\mathbf{q}}}\right) \\
& \mathbf{f}_{\boldsymbol{\rho}}=\frac{\partial \mathbf{f}}{\partial \boldsymbol{\rho}}=\frac{\partial \mathbf{f}_{g r a}}{\partial \boldsymbol{\rho}}-\frac{\partial \mathbf{K}}{\partial \boldsymbol{\rho}}-\frac{\partial \mathbf{C}}{\partial \boldsymbol{\rho}}+\frac{\partial \mathbf{f}_{i n t}}{\partial \boldsymbol{\rho}}
\end{aligned}
$$

As indicated in Section 3.4, is the projection matrix between $\mathbf{f}_{\text {ext }}$ and $\boldsymbol{v}_{\text {ext }}$ at most a linear function of the generalized coordinates. Resulting in $\frac{\partial \mathbf{f}_{e x t}}{\partial \mathbf{q}}$ at most constant and $\frac{\partial \mathbf{f}_{e x t}}{\partial \dot{\mathbf{q}}}$ being zero. Moreover $\frac{\partial \mathbf{K}}{\partial \mathbf{q}}$ is eliminated, similar to using FFRF, but e.g. not with GCMS. Furthermore, no derivatives of the quadratic velocity terms must be computed, which would be the case when using FFRF.

\subsubsection{Effect of FNCF on the generalized internal force}

This section elaborates the computation of the internal loads, the projections to obtain the generalized internal force vector and the accompanied partial derivatives. The simple kinematics of FNCF, described in Section 3.1, leads to first order partial derivatives $\frac{\partial \mathbf{R}}{\partial q_{i}}$ and $\frac{\partial \mathbf{u}}{\partial q_{i}}$ which are constant, and thus second order partial derivatives which are eliminated.

$$
\begin{aligned}
\frac{\mathrm{d}}{\mathrm{dt}}\left(\frac{\partial \mathbf{R}}{\partial \mathrm{q}_{\mathrm{i}}}\right) & =\sum_{n} \frac{\partial^{2} \mathbf{R}}{\partial \mathrm{q}_{\mathrm{i}} \partial \mathrm{q}_{\mathrm{n}}} \dot{\mathrm{q}}_{\mathrm{n}}=0 \\
\frac{\partial^{2} \mathbf{R}}{\partial \mathrm{q}_{\mathrm{j}} \partial \mathrm{q}_{\mathrm{i}}} & =0
\end{aligned}
$$

$$
\begin{aligned}
\frac{\partial^{2} \mathbf{u}}{\partial \mathrm{q}_{\mathrm{j}} \partial \mathrm{q}_{\mathrm{i}}} & =0 \\
\frac{\partial \dot{\mathbf{u}}}{\partial \mathrm{q}_{\mathrm{i}}} & =0
\end{aligned}
$$

Furthermore, these properties also reduce the complexity of computing the Jacobians $\frac{\partial \mathrm{g}}{\partial \mathbf{q}}, \frac{\partial \mathrm{g}}{\partial \dot{\mathbf{q}}}, \frac{\partial \mathrm{g}}{\partial \ddot{\mathbf{q}}}, \frac{\partial \mathrm{g}}{\partial \lambda}$ and $\frac{\partial \mathrm{g}}{\partial \boldsymbol{\rho}}$, especially of objective functions incorporating position, velocity or acceleration measurements.

This section analysed the main advantages of using FNCF for sensitivity analysis using AVM. The implementation is less error-prone, and there is a reduction in computational complexity as various terms and accompanying derivatives can be easier obtained. However, as discussed in Section 3 , the disadvantage of using FNCF is an increased amount of 
generalized coordinates due to the redundant flexible coordinates. This means that the adjoint equations must be solved for more adjoint variables, because there are as many adjoint variables as generalized coordinates. This means a more computational demanding matrix factorization during implicit time integration, and an increase in data-storage needs for the state vector and adjoint variables.

The authors focus on obtaining the various terms in the adjoint equations analytically, because this is often the most efficient method. Reverse automatic differentiation can serve as a good alternative, especially when it is difficult to come up with analytical expressions for these terms. In this way the adjoint variable method and reverse automatic differentiation are used in a combined way. Note that this results in a large reduction of memory usage with respect to the use of reverse automatic differentiation discussed in Section 2.1.3, where the forward simulation code is differentiated.

\section{Application case: MacPherson suspension}

This methodology has been illustrated on a MacPherson suspension test rig shown in Fig.1. The authors want to emphasize that the goal of this demonstrator is not of finding the most accurate model for the suspension strut, but to illustrate that this methodology is an efficient approach for identifying a significant number of parameters that minimizes a prescribed objective function, and this in a general multibody model setting.

\subsection{Test rig and parametrized model}

The test rig is connected to a frame, which is assumed to be rigid, at the locations where the suspension is connected to the chassis of a car. The load enters the system through the tire contact patch, which is excited by a 6 degree of freedom (DOF) hydraulic shaker, as shown in figure 1.

The MacPherson suspension test rig consists of 5 bodies which are the control arm, steering knuckle, tierod, lower strut and upper strut. The steering knuckle and control arm are considered to be flexible components. Both components are reduced using component mode synthesis [17] by the Craig-Bampton technique [18]. The steering knuckle is then connected to the lower strut by a rigid joint and to both the tierod and the control arm by two additional spherical joints. Finally, the lower strut and upper strut are connected to each other by a cylindrical joint. The system has hence one global degree of freedom corresponding to the global vertical motion. The MacPherson suspension and its model are given in the Fig.1.

The suspension strut is composed of a spring and damper. The spring is modelled using a linear spring element with stiffness $k$ and free length $L$. The damper is modelled by a viscous friction $f_{v f}$ in combination with a nonlinear friction model $f_{n f}$ :

$$
f_{\text {strut }}=k(x-L)+f_{v f}+f_{n f}
$$

with the model parameter $c$ representing the viscous friction part and parameters $a_{f}, b_{f}, c_{f}$ and $e_{f}$ representing the nonlinear friction part that is based on the Pacejka formula [19]. Furthermore, the latter five parameters can differ during the compression-rebound stroke of the suspension. This is indicated with a subscript ${ }_{1}$ for rebound and ${ }_{2}$ for compression:

$$
\left\{\begin{array} { l l } 
{ f _ { v f } = c _ { 1 } v } & { \text { if } \mathrm { v } \geq 0 } \\
{ f _ { v f } = c _ { 2 } v } & { \text { if } \mathrm { v } < 0 }
\end{array} ( 2 2 \mathrm { b } ) \quad \left\{\begin{array}{ll}
f_{n f}=a_{f_{1}} \sin \left(c_{f_{1}} \tan ^{-1}\left[b_{f_{1}}\left(1-e_{f_{1}}\right) v+e_{f_{1}} \tan ^{-1}\left(b_{f_{1}} v\right)\right]\right) & \text { if } \mathrm{v} \geq 0 \\
f_{n f}=a_{f_{2}} \sin \left(c_{f_{2}} \tan ^{-1}\left[b_{f_{2}}\left(1-e_{f_{2}}\right) v+e_{f_{2}} \tan ^{-1}\left(b_{f_{2}} v\right)\right]\right) & \text { if } \mathrm{v}<0 .
\end{array}\right.\right.
$$

For the simulation model, the external loads are considered to be applied at the wheel center (WC) which is a point rigidly attached to the knuckle and is also highlighted in Fig.1. These external loads consist of three forces and three moments along and about the three global axes. The applied WC loads are obtained from a load cell mounted on the experimental setup, as shown in Fig.1. The load cell is interposed between the knuckle and the tire rim hence it directly measures the loads providing the input signals applied to the model. While being rigidly connected on the knuckle side, the load cell includes bearings as well as a rotating spindle on the tire rim side such that the rolling motion of the tire is allowed.

The objective function is based on the spring-damper deflection measurements obtained from a displacement sensor, shown on Fig.1. The deflection is initialized to zero at the start of the measurement campaign. Because of this initialization, an offset value $y_{0}$ is subtracted from the simulated spring deflection that computes the absolute distance between the two measurement points:

$$
g=\left(\left(\left[\begin{array}{lll}
0 & 0 & 1
\end{array}\right] \mathbf{R}^{\mathrm{T}}\left(\mathbf{u}_{2}-\mathbf{u}_{1}\right)-y_{0}\right)-\hat{y}(t)\right)^{2},
$$

with $\mathbf{R}$ the rotation matrix of a local coordinate system that has the z-axis aligned with the suspension strut. This matrix transforms the position difference of the spring ends positions $\mathbf{u}_{2}$ and $\mathbf{u}_{1}$ from a global reference frame to the local axis 

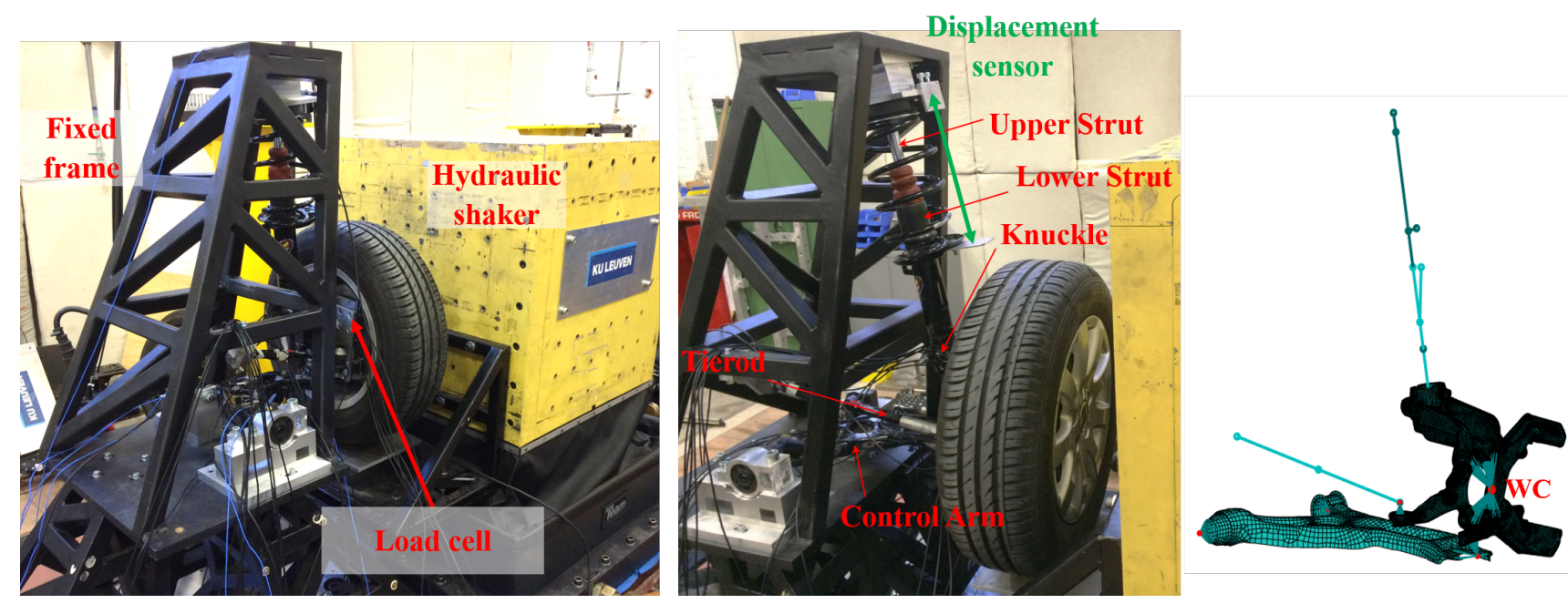

Fig. 1: Pictures of the test setup, and on the right the corresponding model [20]

frame. Furthermore, since the offset value $y_{0}$ is unknown, it will be identified as well during the parameter identification process.

\subsection{Practical considerations in identification setup}

The optimizer that has been used is IPopt [21]. This optimizer uses the interior-point algorithm and uses the BFGS method [4] for the Hessian approximation.

Scaling has been applied in order to prevent numerical issues. First, the residual of the objective function which is obtained using the initial guess of the parameters, is used to normalize the residuals during the optimization. Furthermore, this scaling would be applied to all measurement quantities in case more than one would be used. This results in the possibility to treat different measurement quantities the same way in one objective function. Using weighting factors it is then possible to give more importance to specific measurement quantities. Second, the model parameters are scaled with $s_{i}$. This scaling value is based on the span of the parameter's specific upper and lower bound interval, denoted by $\rho_{i}^{\max }$ and $\rho_{i}^{\min }$ respectively. : $s_{i}=\frac{2}{\left(\rho_{i}^{\max }-\rho_{i}^{m i n}\right)}$.

\subsection{Identification framework}

Figure 2 illustrates the process of parameter identification on the MacPherson suspension, using the adjoint variable method for sensitivity analysis. The input to the test rig is the displacement of the shaker, that lifts the tire up and down. The load cells measures the resulting forces at the wheel center. These dynamic loads are then used as an input to the model. First the initial conditions $[\mathbf{q}, \dot{\mathbf{q}}, \ddot{\mathbf{q}}, \boldsymbol{\lambda}]_{T_{0}}$ and its derivatives $\left[\frac{\mathrm{d} \mathbf{q}}{\mathrm{d} \boldsymbol{\rho}}, \frac{\mathrm{d} \dot{\mathbf{q}}}{\mathrm{d} \boldsymbol{\rho}}\right]_{T_{0}}$ are computed. Afterwards the equations of motions are solved and the residual of the objective function is computed. Next, the gradient computation starts with determining $\left[\boldsymbol{\mu}, \dot{\boldsymbol{\mu}}, \ddot{\boldsymbol{\mu}}, \boldsymbol{\mu}_{\boldsymbol{\phi}}\right]_{T_{F}}$ by solving Eq.(17d), followed by the backward integration of the adjoint equations. This integration process uses the solution of the forward simulation, $\mathbf{q}, \dot{\mathbf{q}}, \ddot{\mathbf{q}}, \boldsymbol{\lambda}$, and functionality of the multibody model in order to compute the terms appearing in Eq.(16). The sensitivity is computed using Eq.(18) and passed on to the optimizer. If the tolerance criteria of the optimizer are not met, which means that the optimizer did not converge, a new iteration starts with a new guess $\boldsymbol{\rho}^{*} \in \mathbb{R}^{n_{\rho}}$ of the model parameters. If the optimizer converges to a local minimum, it will output the converged parameter values.

The constraint equations $\boldsymbol{\phi}$ are for this application independent of the model parameters $\boldsymbol{\rho}$. Furthermore, the least squares objective function, defined in Eq.(23), only depends on the generalized coordinates $\mathbf{q}$ and the model parameters $\boldsymbol{\rho}$. Moreover, the system mass matrix $\mathbf{M}$ is also independent of the model parameters. The expression for obtaining the sensitivity information, given in Eq. (18), can then be condensed to:

$$
\nabla_{\boldsymbol{\rho}} \psi^{\mathrm{T}}=\left[\left(-\dot{\boldsymbol{\mu}}^{\mathrm{T}} \mathbf{M}-\boldsymbol{\mu}^{\mathrm{T}} \frac{\partial \mathbf{f}}{\partial \dot{\mathbf{q}}}\right) \frac{\mathrm{d} \mathbf{q}}{\mathrm{d} \boldsymbol{\rho}}\right]_{T_{0}}-\left[\left(-\boldsymbol{\mu}^{\mathrm{T}} \mathbf{M}\right) \frac{\mathrm{d} \dot{\mathbf{q}}}{\mathrm{d} \boldsymbol{\rho}}\right]_{T_{0}}+\int_{T_{0}}^{T_{F}}\left(\frac{\partial \mathrm{g}}{\partial \boldsymbol{\rho}}-\boldsymbol{\mu}^{\mathrm{T}}\left(-\frac{\partial \mathbf{f}}{\partial \boldsymbol{\rho}}\right)\right) \mathrm{dt}
$$

\subsection{Results}

Table 1 shows the initial guess, the lower and upper bounds and the optimized value of the model parameters. Furthermore, the table also shows the sensitivity information using finite differences, the adjoint variable method for the first 


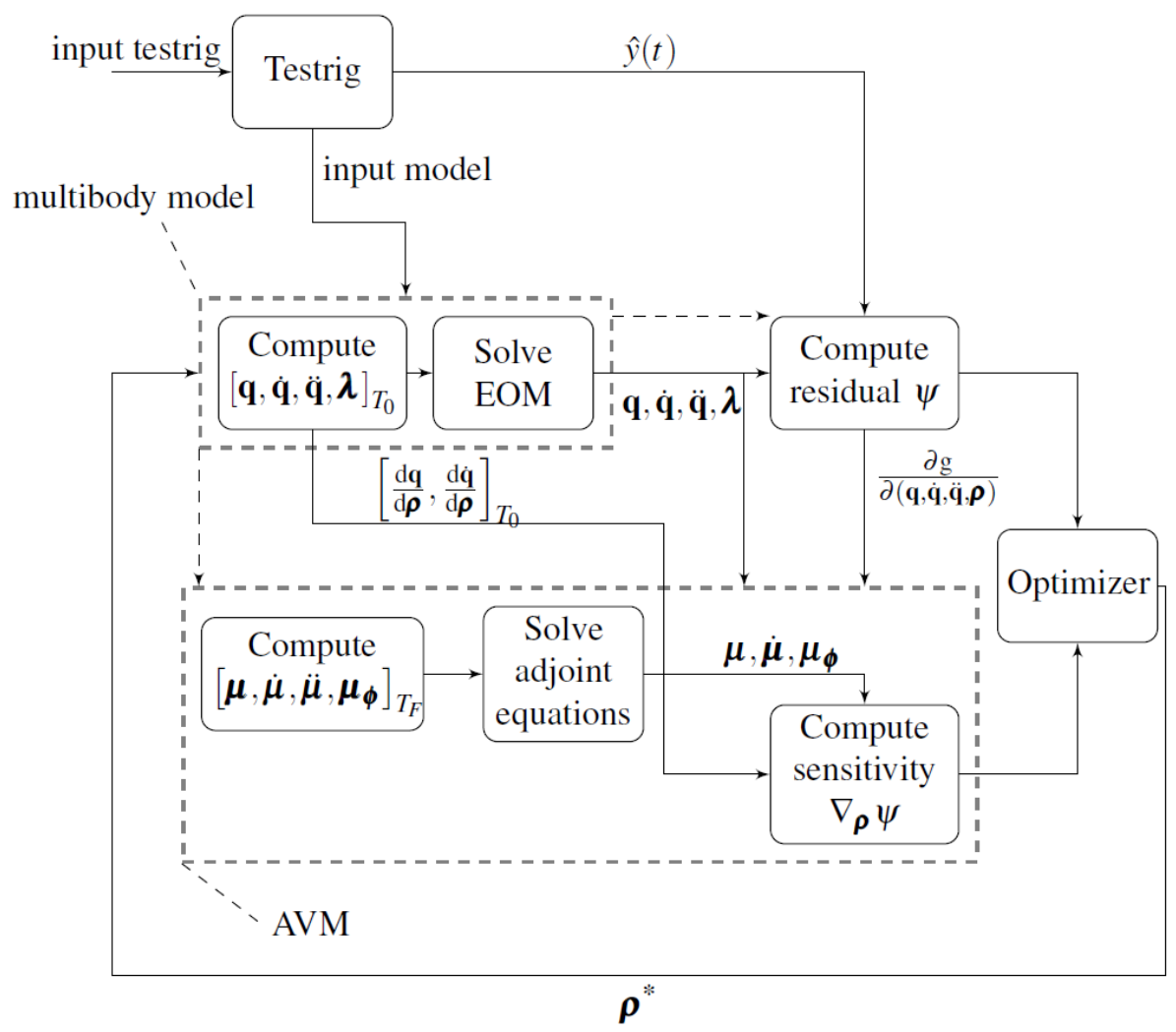

Fig. 2: Parameter identification framework

Table 1: Startvalues and optimized values of model parameters. Sensitivity analysis using finite differences and AVM at start iteration process

\begin{tabular}{|c|cccc|ccc|}
\hline Parameter & Initial value & Lower bound & Upper bound & Optimized value & FD result & AVM & ratio \\
\hline$k$ & 23500 & 22000 & 24000 & 23999.75 & $-3.9307 \mathrm{E}-3$ & $-3.9285 \mathrm{E}-3$ & 1.0006 \\
$L$ & 0.343 & 0.34 & 0.35 & 0.3424 & $-7.1227 \mathrm{E} 2$ & $-7.1178 \mathrm{E} 2$ & 1.0007 \\
$c_{1}$ & 2000 & 500 & 5000 & 2344.78 & $8.6009 \mathrm{E}-4$ & $8.6038 \mathrm{E}-4$ & 0.9997 \\
$c_{2}$ & 3000 & 500 & 5000 & 3116.36 & $-6.9682 \mathrm{E}-4$ & $-6.9883 \mathrm{E}-4$ & 0.9971 \\
$a_{f_{1}}$ & 100 & 0 & 500 & 67.99 & $1.4470 \mathrm{E}-2$ & $1.4414 \mathrm{E}-2$ & 1.0039 \\
$b_{f_{1}}$ & 50 & 10 & 2000 & 71.33 & $1.9617 \mathrm{E}-3$ & $1.9871 \mathrm{E}-3$ & 0.9871 \\
$c_{f_{1}}$ & 1.2 & 1 & 1.9 & 1.888 & $-3.1490 \mathrm{E}-1$ & $-3.1642 \mathrm{E}-1$ & 0.9952 \\
$e_{f_{1}}$ & -10 & -50 & 1 & -12.35 & $-1.4108 \mathrm{E}-3$ & $-1.4092 \mathrm{E}-3$ & 1.0011 \\
$a_{f_{2}}$ & 100 & 0 & 500 & 214.17 & $-1.3007 \mathrm{E}-2$ & $-1.2994 \mathrm{E}-2$ & 1.0009 \\
$b_{f_{2}}$ & 50 & 10 & 2000 & 68.17 & $-9.7895 \mathrm{E}-4$ & $-9.7673 \mathrm{E}-4$ & 1.0022 \\
$c_{f_{2}}$ & 1.2 & 1 & 1.9 & 1.395 & $3.3045 \mathrm{E}-1$ & $3.3193 \mathrm{E}-1$ & 0.9955 \\
$e_{f_{2}}$ & -10 & -50 & 1 & -11.89 & $1.9807 \mathrm{E}-4$ & $1.9795 \mathrm{E}-4$ & 1.0006 \\
$y_{0}$ & -0.21445 & -0.22 & -0.21 & -0.21650 & $-7.8054 \mathrm{E} 2$ & $-7.8058 \mathrm{E} 2$ & 0.9999 \\
\hline
\end{tabular}

iteration, and the ratio of the sensitivity analysis using AVM with respect to finite differences. Different perturbation sizes need to be applied in order to obtain reliable finite difference sensitivities. This table demonstrates the validity of the sensitivity information obtained through the AVM approach with underlying FNCF. As the spring stiffness $k$, its free length $L$ and the offset value $y_{0}$ were known parameters, the upper and lower bounds span a relative small interval.

Figure 3 shows the spring deflection during a time interval of three seconds. Three lines are shown, which are the measured deflection of the spring, the result of the simulation using the initial values of the optimization and the simulation using the optimized values. This figure clearly demonstrates the improvement in model fit as a result of the optimized model parameters. Moreover, it is clear that the current model structure does not allow to simulate the (nonlinear) effects 


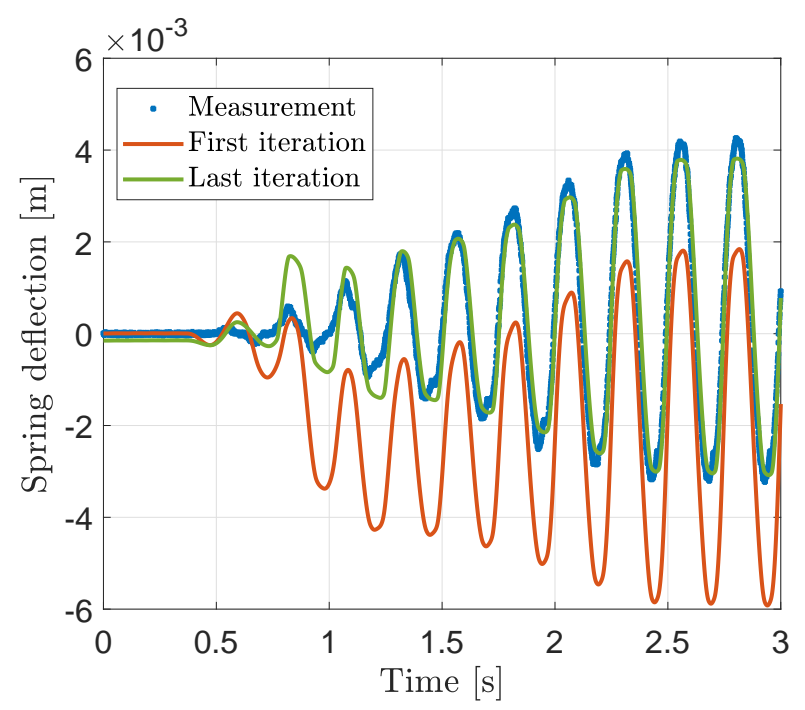

Fig. 3: Spring deflection of the measurement, the simulation using the initial guess and the optimized model parameters

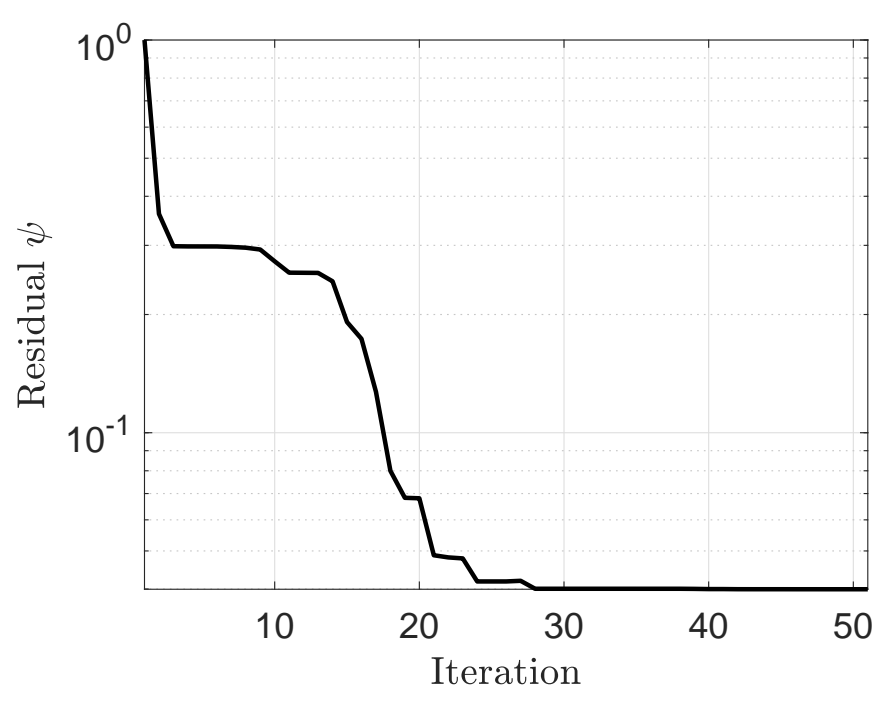

Fig. 4: Variation of the residual of the objective function during optimization

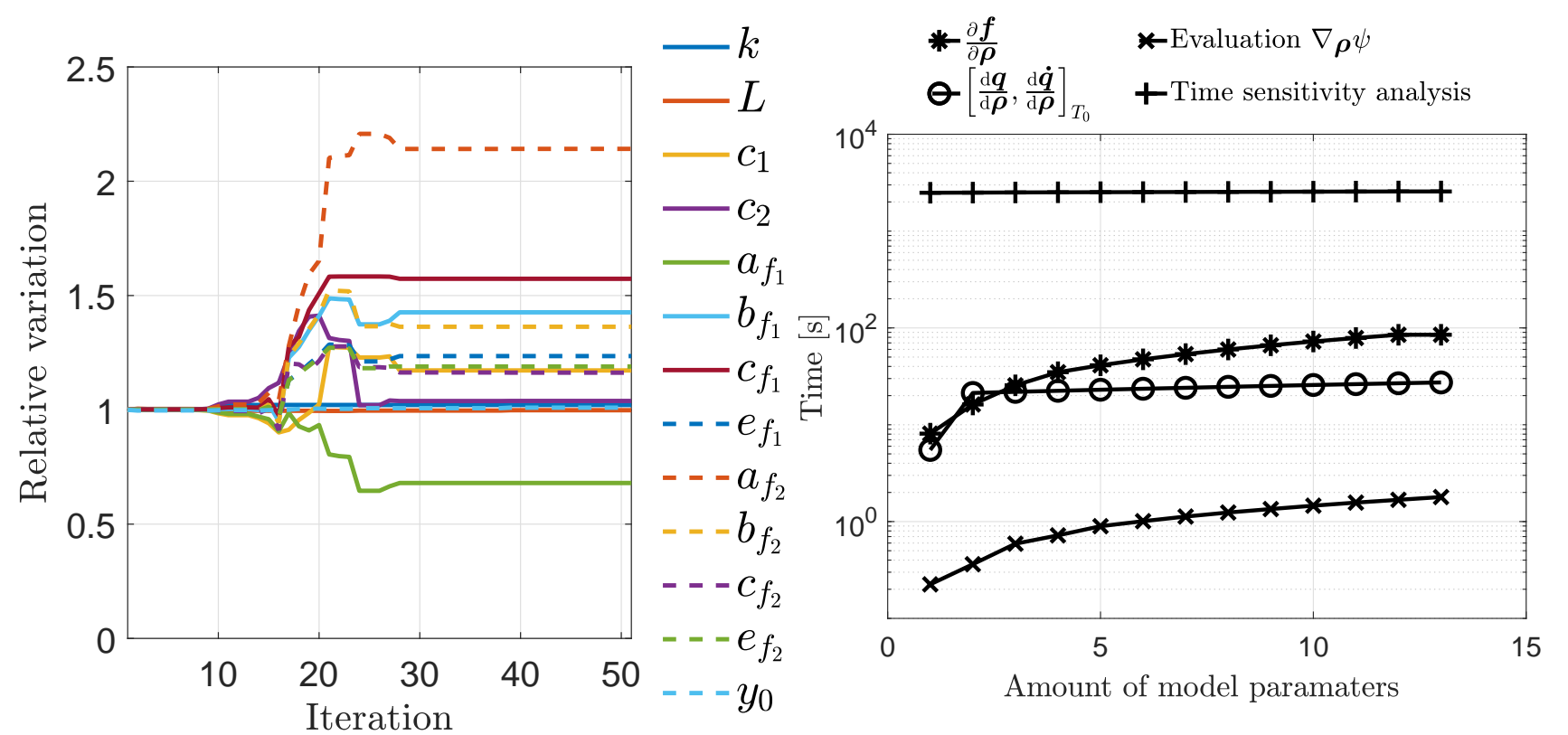

Fig. 5: Relative parameter variation during the optimization

Fig. 6: Effect of increasing amount of model parameters on computation time

Figure 4 shows the residual during the optimization. As discussed in Section 5.2, the residual is normalized at the start of the optimization routine.

Figure 5 shows the variation of the model parameters during the optimization, relative to the initial values. The optimization process was terminated after 51 iterations, where the parameter variation was below the specified threshold value. Both Fig.4 and Fig.5 demonstrate rapid and consistent convergence using the AVM approach in the multibody model, even over significant parameter variations.

Figure 6 illustrates the property of the AVM that the total computation time for the sensitivity information is relatively invariant of the model parameters. The terms in Eq.(24) that do scale with the amount of model parameters are $\left[\frac{\mathrm{d} \mathbf{q}}{\mathrm{d} \boldsymbol{\rho}}, \frac{\mathrm{d} \dot{\mathbf{q}}}{\mathrm{\rho} \boldsymbol{\rho}}\right]_{T_{0}}$, 
$\frac{\partial \mathbf{f}}{\partial \boldsymbol{\rho}}$ and $\frac{\partial \mathrm{g}}{\partial \boldsymbol{\rho}}$. In this application, the latter term is only different from zero for the $y_{0}$ parameter and is therefore not included in the figure. Furthermore, its evaluation time was only $15.5 \mathrm{~ms}$. It must be noted that the computation of $\left[\frac{\mathrm{d} \mathbf{q}}{\mathrm{d} \boldsymbol{\rho}}, \frac{\mathrm{d} \dot{\mathbf{q}}}{\mathrm{\rho} \boldsymbol{\rho}}\right]_{T_{0}} \mathrm{does}$ not scale with the length of the time signal, however evaluating the integral and the computation of $\frac{\partial \mathbf{f}}{\partial \boldsymbol{\rho}}$ does scale with the length of the time signal used for the optimization. Although, the cost is relatively small compared to the time needed for backward integration of the adjoint equations. Overall in this case, for each iteration, we observed a computational load of, on average, three times the load for a single forward simulation, close to independent of the number of model parameters to identify. This demonstrates the large power of the AVM approach combined with the FNCF modelling scheme in order to tackle practical application where numerous parameters typically need to be determined.

\section{Conclusions}

This work proposes a technique for the in-situ identification of parameters of flexible multibody systems using systemlevel measurements. The sensitivity information is obtained using the adjoint variable method, of which the evaluation of the terms in the adjoint equations benefit from the advantageous properties of the underlying Flexible Natural Coordinates Formulation.

The proposed approach of using FNCF results in a significant decrease in the implementation and computational complexity with respect to alternative approaches based on other multibody formulations, as the various terms in the equations of motion and their partial derivatives can be readily obtained and are of limited order. A trade-off for the reduction of computational complexity is the increased amount of generalized coordinates due to the redundant flexible coordinates present in the FNCF method. This means that the equations of motion and adjoint equations must be solved for more unknowns, what translates in a more demanding matrix factorization during implicit time integration, and an increase in storage needs for the state vector and adjoint variables.

The proposed methodology is demonstrated on a MacPherson suspension test rig where thirteen model parameters have been identified that minimized the spring deflection difference between measurements and simulation.

\section{Acknowledgements}

The research of S. Vanpaemel and F. Naets is funded by a grant from the Research Foundation - Flanders (FWO). The authors acknowledge dr. ir. E. Risaliti for providing the multibody model and measurement data of the suspension test rig.

\section{References}

[1] Dzierzek, S., 2000. "Experiment-based modeling of cylindrical rubber bushings for the simulation of wheel suspension dynamic behavior". SAE transactions, 109(6), pp. 78-85.

[2] Berg, M., 1998. “A Non-Linear Rubber Spring Model for Rail Vehicle Dynamics Analysis”. Vehicle System Dynamics, 30(3-4), Sept., pp. 197-212.

[3] Sedlaczek, K., Dronka, S., and Rauh, J., 2011. "Advanced modular modelling of rubber bushings for vehicle simulations”. Vehicle System Dynamics, 49(5), May, pp. 741-759.

[4] Nocedal, J., and Wright, S. J., 2006. Numerical optimization, 2nd ed. Springer series in operations research. Springer, New York. OCLC: ocm68629100.

[5] Serban, R., and Freeman, J. S., 2001. "Identification and Identifiability of Unknown Parameters in Multibody Dynamic Systems”. Multibody System Dynamics, 5(4), May, pp. 335-350.

[6] Haug, E. J., 1987. "Design Sensitivity Analysis of Dynamic Systems". In Computer Aided Optimal Design: Structural and Mechanical Systems, NATO ASI Series. Springer, Berlin, Heidelberg, pp. 705-755.

[7] Bestle, D., and Eberhard, P., 1992. "Analyzing and Optimizing Multibody Systems". Mechanics of Structures and Machines, 20(1), Jan., pp. 67-92.

[8] Nachbagauer, K., Oberpeilsteiner, S., Sherif, K., and Steiner, W., 2015. "The Use of the Adjoint Method for Solving Typical Optimization Problems in Multibody Dynamics". Journal of Computational and Nonlinear Dynamics, 10(6), p. 61011.

[9] Bischof, C. H., 1996. "On the Automatic Differentiation of Computer Programs and an Application to Multibody Systems". In IUTAM Symposium on Optimization of Mechanical Systems, Solid Mechanics and its Applications. Springer, Dordrecht, pp. 41-48.

[10] Vermaut, M., Naets, F., and Desmet, W., 2018. "A flexible natural coordinates formulation (FNCF) for the efficient simulation of small-deformation multibody systems". International Journal for Numerical Methods in Engineering, 115(11), pp. 1353-1370.

[11] Schmidt, T., and Müller, P. C., 1993. "A parameter estimation method for multibody systems with constraints". In Advanced Multibody System Dynamics, W. Schiehlen, ed., Vol. 20. Springer, Dordrecht, pp. 427-432. 
[12] Ayusawa, K., Venture, G., and Nakamura, Y., 2014. "Identifiability and identification of inertial parameters using the underactuated base-link dynamics for legged multibody systems". The International Journal of Robotics Research, 33(3), pp. 446-468.

[13] Haug, E. J., ed., 1993. Concurrent Engineering: Tools and Technologies for Mechanical System Design. Springer Berlin Heidelberg, Berlin, Heidelberg.

[14] Zhu, Y., 2014. "Sensitivity Analysis and Optimization of Multibody Systems". PhD thesis, Virginia Polytechnic Institute and State University.

[15] Shabana, A. A., 2013. Dynamics of Multibody Systems, 4 ed. Cambridge University Press.

[16] Pechstein, A., Reischl, D., and Gerstmayr, J., 2013. "A generalized component mode synthesis approach for flexible multibody systems with a constant mass matrix". Journal of Computational and Nonlinear Dynamics, 8(1), p. 011019.

[17] Hurty, W. C., 1965. "Dynamic analysis of structural systems using component modes". AIAA Journal, 3(4), pp. 678685.

[18] Craig, R., and Bampton, M., 1968. "Coupling of Substructures for Dynamic Analyses". AIAA Journal, American Institute of Aeronautics and Astronautics, 6(7), pp. 1313-1319.

[19] Pacejka, H. B., and Bakker, E., 1992. "The magic formula tyre model”. Vehicle System Dynamics, 21(sup001), pp. 118.

[20] Risaliti, E., Tamarozzi, T., Vermaut, M., Cornelis, B., and Desmet, W., 2019. "Multibody model based estimation of multiple loads and strain field on a vehicle suspension system". Mechanical Systems and Signal Processing, 123, pp. 1-25.

[21] Wächter, A., and Biegler, L. T., 2006. "Digital Object Identifier ( On the implementation of an interior-point filter line-search algorithm for large-scale nonlinear programming”. Math. Program., Ser. A, 106, pp. 25-57. 